\title{
Differentiating Vogt-Koyanagi-Harada syndrome from recurrent optic neuritis: a case report and review of the literature concerning Hispanic patients
}

\author{
Marta Scarioni ${ }^{i^{* \dagger}} \mathbb{D}$, Anna M. Pietroboni ${ }^{1 \dagger}$, Alessandro Invernizzi ${ }^{2,3}$, Francesco Viola ${ }^{2}$, Laura Ghezzi ${ }^{1}$, Alberto Calvi ${ }^{1}$,
} Tiziana Carandini ${ }^{1}$, Milena De Riz ${ }^{1}$, Daniela Galimberti ${ }^{1}$ and Elio Scarpini ${ }^{1}$

\begin{abstract}
Background: First recognized at the beginning of twentieth century and named after three authors who independently described some affected patients, Vogt-Koyanagi-Harada syndrome is a rare multisystemic autoimmune disease targeting melanin-containing tissues of the eye, meninges, inner ear and skin. It predominantly affects Asian people, but also people with darker skin pigmentation such as Native Americans and Hispanics (Mestizos), whose ancestors moved from Asia across the Bering strait to North America and further down to Central and South America. Heterogenous presentation is observed, especially among different ethnic groups. Here we describe the case of an Hispanic South American patient presenting with multiple visual relapses and thus mimicking recurrent optic neuritis; we provide insights into the differential diagnosis and a brief review of the literature concerning the epidemiology of Vogt-Koyanagi-Harada syndrome in Hispanic patients compared with other ethnic groups.
\end{abstract}

Case presentation: A 34-year-old Ecuadorian woman presented over years with multiple relapses involving the visual system. She was investigated in both neurologic and ophthalmic clinical settings. Brain Magnetic Resonance Imaging, cerebrospinal fluid examination, Spectral Domain Optical Coherence Tomography and Fluorescein Angiography were performed. She was misdiagnosed first as an optic neuritis pointing to a demyelinating disorder, then as a posterior scleritis. Due to the protean manifestations of Vogt-Koyanagi-Harada syndrome and the incomplete clinical presentation at the beginning, the right diagnosis was made only at a later disease stage using retrospective criteria.

Conclusions: Hispanic patients often present without extraocular symptoms in early phases of the disease and they have globally lower rates of intertegumentary signs compared to Asian patients. The diagnosis of a multisystemic disease such as Vogt-Koyanagi-Harada syndrome is a challenge involving specialists operating in different medical fields; especially in urban multiethnic populations, rare etiologies of common symptoms have to be taken into account when performing a differential diagnosis.

Keywords: Vogt-Koyanagi-Harada syndrome, Epidemiology, Case report, South America, Hispanic, Differential diagnosis, Optic neuritis, Uveitis, Multiple sclerosis

\footnotetext{
* Correspondence: marta.scarioni@gmail.com

${ }^{\dagger}$ Equal contributors

'Department of Pathophysiology and Transplantation, Neurology Unit, Dino

Ferrari Center, University of Milan, Fondazione IRCCS Ca' Granda, Ospedale

Maggiore Policlinico, Milan, Italy

Full list of author information is available at the end of the article
} 


\section{Background}

First recognized at the beginning of twentieth century and named after three authors who independently described some affected patients [1], Vogt-KoyanagiHarada $(\mathrm{VKH})$ is a rare multisystemic autoimmune disease targeting melanin-containing tissues of the eye, meninges, inner ear and skin [2]. It predominantly affects Asian people, but also people with darker skin pigmentation such as Native Americans and Hispanics (Mestizos), whose ancestors moved from Asia across the Bering strait to North America and further down to Central and South America [3, 4]. Genetic susceptibility is linked to certain HLA alleles, the most relevant being HLA-DRB1*0405, which has been reported as the predominant allele across different ethnic groups [5]. The typical age of incidence is between 20 and 50 years, and women are affected twice than men. The most prominent feature of $\mathrm{VKH}$ is bilateral uveitis, accompanied by various combinations of neurological, auditory and cutaneous signs and symptoms. The typical disease course includes four phases (prodromic, uveitic, convalescent and recurrent/chronic), with very protean clinical manifestations [6]. New diagnostic criteria take into account such clinical variability [7].

\section{Case presentation}

A 34-year-old Ecuadorian women was referred to our hospital for sudden loss of vision and pain in her right eye, immediately followed by the same symptoms in the left eye. At presentation bilateral visual impairment with a best corrected visual acuity (BCVA) of 20/200 in the right eye and 20/60 in the left eye was detected. Ophthalmoscopic examination revealed optic nerve head edema without any other sign of inflammation. The patient was hence referred to the neurological ward with a diagnose of "bilateral papilledema - suspected optic neuritis (ON)". Here, she underwent neurological examination, brain and spinal cord Magnetic Resonance Imaging (MRI), lumbar puncture (LP) and autoantibodies testing (anti AQP4 and anti-MOG) to rule out a demyelinating disease of the Central Nervous System (CNS). Neurological examination and MRI resulted within normal limits. Autoantibodies testing was negative. Cerebrospinal fluid (CSF) examination showed a mild pleocytosis in the absence of oligoclonal bands (OCBs). She was treated with a short-term steroid therapy and discharged with the diagnosis of "bilateral ON with liquor pleocytosis".

Six years later, another episode of sudden loss of vision and pain in the left eye occurred, and the patient was admitted to the ophthalmology ward for worsening symptoms. She underwent a complete ophthalmological examination, including Spectral Domain Optical Coherence Tomography (SD-OCT) and Fluorescein Angiography
(FA). SD-OCT revealed a large exudative retinal detachment (ERD) at the posterior pole in the left eye and bilateral choroidal folds (Fig. 1). FA showed optic disc hyperfluorescence during late phases of the angiogram as well as multiple areas of leakage in the left eye (Fig. 2). A work-up for posterior uveitis was hence performed. Blood tests for infectious diseases commonly causing uveitis (herpetic viruses, syphilis, borreliosis, tuberculosis) were negative as well as autoantibodies (ANA, ASMA, ANCA, anti-dsDNA, ENA) screening. A complete neurological evaluation was then repeated; this time CSF examination was normal, with neither pleocytosis nor OCBs, while MRI of the eyes and orbits revealed a scleroretinal thickening with enhancement in the posterior chamber of the left eye. According to these findings the disease was labelled as "probable posterior scleritis (PS)". A three-days high-dose intravenous steroid treatment was started, followed by slow oral taper in the following months. The patient improved both clinically and instrumentally.

A year and a half later, the patient came to our attention complaining of loss of vision in the right eye, accompanied by headache, neck stiffness, slight subjective left hearing loss and tinnitus. At funduscopic examination, optic nerve head edema was detected in the right eye (Fig. 3). The relapsing nature of the condition along with the protean clinical manifestations of the disease through the years raised the suspect of VKH disease. The medical history of the patient was hence retrospectively investigated and the CSF pleocytosis identified at the first presentation was finally correctly reinterpreted. In addition to those described, the patient reported other preceding episodes of visual loss diagnosed elsewhere as ONs and treated with intravenous steroid therapy followed by slow oral taper. There was no laboratory evidence suggestive of other autoimmune or infective ocular disease entities, nor a previous history of penetrating ocular trauma or surgery. According to revised diagnostic criteria for VKH disease (Read et al., 2001), a diagnosis of incomplete VKH was finally made. She was treated with oral steroid therapy, with partial edema resolution on a 3-months eye examination. A long-term treatment with Azathioprine was then started.

In order to support our diagnosis, a HLA haplotype typization was performed. However, HLA-DRB1*0405 allele was absent.

\section{Discussion and conclusions}

Here, we describe a case of $\mathrm{VKH}$, whose diagnosis was done retrospectively due to the protean manifestations of this condition. The first presentations, including loss of vision accompanied by pain in the eye, suggested in fact $\mathrm{ON}$ [8] or posterior scleritis (PS) [9]. 


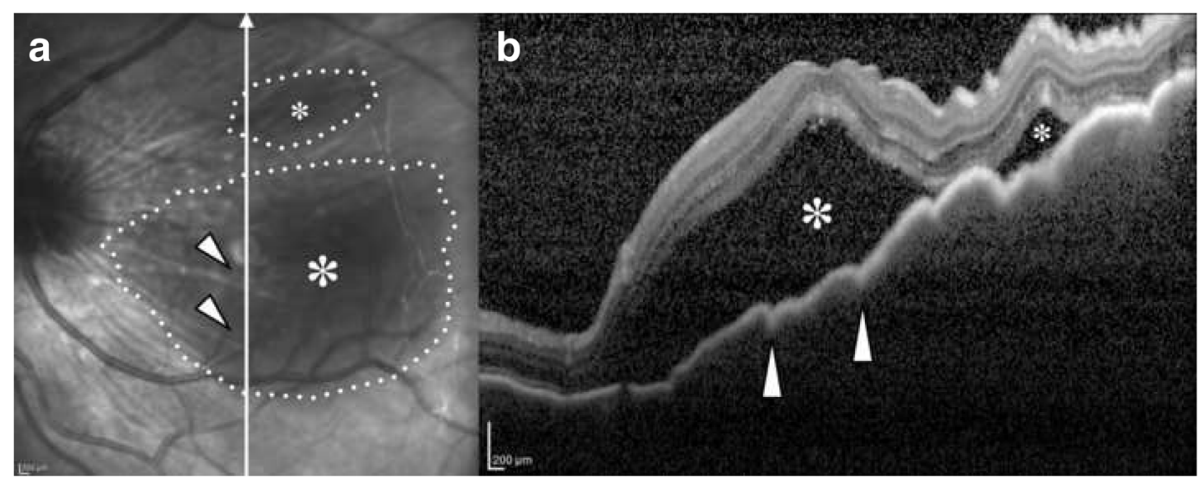

Fig. 1 Combined Near Infrared Reflectance (NIR) (a) and Spectral Domain Optical Coherence Tomography (SD-OCT) (b) of the left eye performed during a disease recurrence occurred six years after the first presentation. The white arrow crossing NIR image corresponds to the position of the SD-OCT scan. Multiple pockets of fluid accumulation (white asterisk) detaching the neuroretina are visible as dark gray areas (encircled by white dotted lines) on NIR and as empty spaces on SD-OCT. Choroidal folds are also clearly visible in both the images (white arrowheads)

The first clinical episode was investigated in a neurological setting, with optic nerve head edema as solely clinical finding leading to high suspicion of $\mathrm{ON}$ as a Clinically Isolated Syndrome (CIS) pointing to a demyelinating disorder such as Multiple Sclerosis (MS) or, given the bilateral and exclusive involvement of the visual system, a Neuromyelitis Optica Spectrum Disorder (NMOSD) [10]; little attention was hence given to liquoral pleocytosis in the absence of OCBs, since both these findings were still compatible with a CNS demyelinating disease. According to the misdiagnosis, a suboptimal dose of steroid was administered at that time, which can at least partially explain the subsequent propensity for recurrence [11].

The second clinical episode was investigated by ophthalmologists. On that occasion clinical and instrumental findings (OCT, FA) could rise the suspicion of both a PS and a VKH $[12,13]$, but the absence of CSF pleocytosis (probably due to prior long-term steroid administration) did not support the latter hypothesis, which seemed also the less likely from a purely epidemiological point of view.

The third clinical episode manifested with polymorphic symptoms suggesting a VKH syndrome and the patient was evaluated in a multidisciplinary setting, so that the right diagnosis was made at a later disease stage using retrospective criteria.

The lack of communication between specialists operating in different medical fields can lead to a significant diagnostic delay, especially with rare multisystemic diseases. Crossing knowledge boundaries, which are often not clearly defined in such disorders, is essential to reach
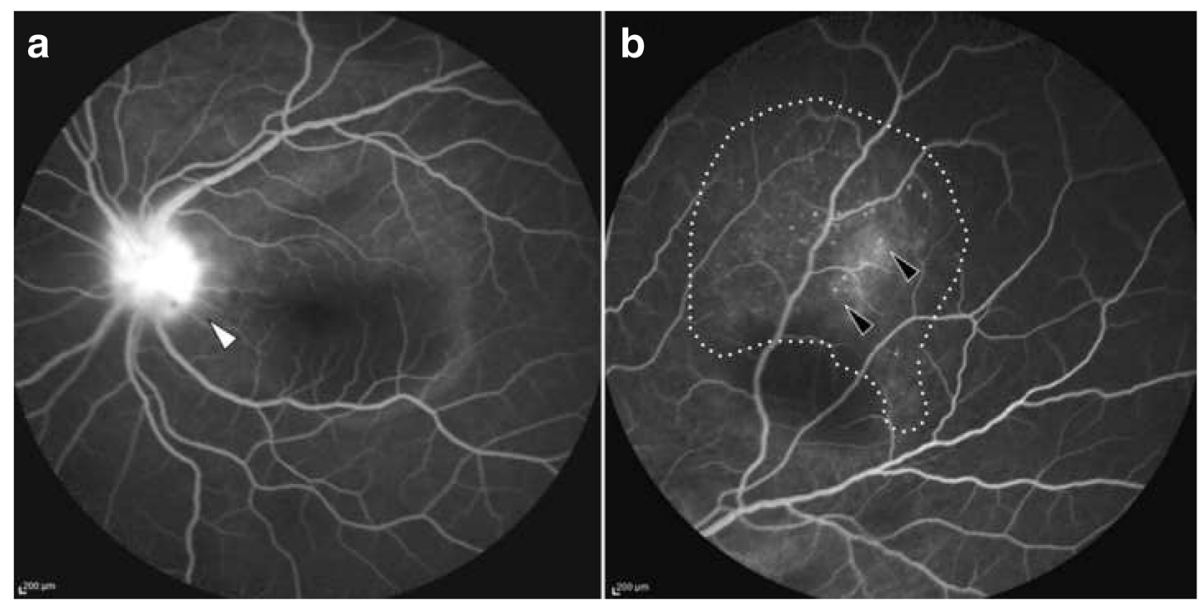

Fig. 2 Fluorescein angiography (FA) of the left eye performed during a disease recurrence occurred six years after the first presentation. Images of the posterior pole (a) during the late phases of the angiogram reveal leakage of dye from the optic nerve head (white arrowhead) demonstrating the inflammatory status. Multiple leaking points (black arrowheads) feeding a pocket of subretinal fluid (encircled by white dotted lines) are visible along the supero-temporal vascular arcade (b) 


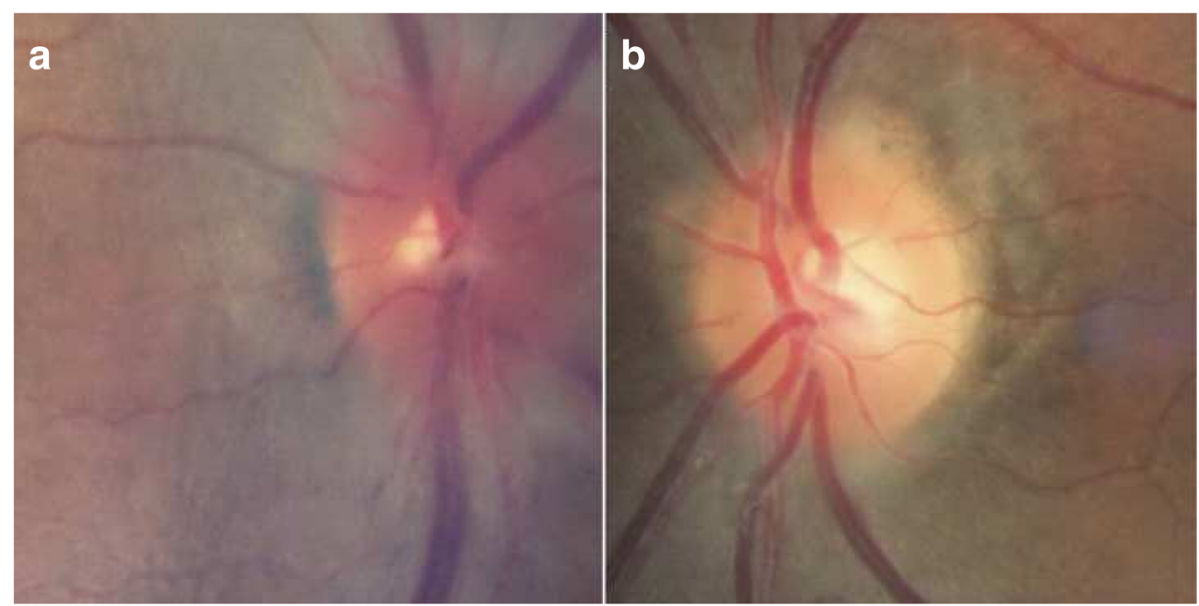

Fig. 3 Fundus photography of both eyes (optic nerve head field) performed during the last disease recurrence. Optic nerve head appears hyperemic and swollen in the right eye (a) as compared to the left eye (b) where the disc looks within normal limits

the goal of early diagnosis and treatment which, in turn, significantly affects prognosis, avoiding long-term complications such as severe iris depigmentation and persistent ocular hypotony, which have been described in South American patients [14].

VKH is a rare disease in Europe. In Switzerland, where the first patient was described by Vogt [5], VKH represents $0.7 \%$ of all uveitis [15]. Two studies from the North of Italy, where our hospital is located, report VKH as accounting for $1,37-4,1 \%$ of all uveitis (the former being a percentage even lower then the rest of Europe) $[16,17]$. VKH is mostly seen (and thus, suspected) among patients of Asian origin. In China the highest percentage of panuveitis is reported [18], due to the high incidence of both $\mathrm{VKH}$ and Bechet disease (accounting respectively for $29-30 \%$ of all uveitis) [19]. In Japan VKH was considered the first cause of uveitis $[18,20]$, but differences in etiology of uveitis are being observed over time [21], and in most recent studies it is reported as the second cause of uveitis (7\% of all uveitis) after sarcoidosis $[22,23]$. On the other hand, higher VKH rates than expected are being recorded in South America: in Chile [24] VKH has been reported as the most frequent cause of uveitis, accounting for $17,2 \%$ of all uveitis, and in Argentina as the second cause after toxoplasmosis, accounting for $16.7 \%$ of all uveitis [18]. An epidemiologic study on uveitis conducted in Barcelona and highlighting the challenge of globalization in urban multiethnic populations reported a South American ethnicity in $47 \%$ of cases of VKH [25].

Ethnic groups do not only differ with respect to incidence and prevalence of the disease, but also for the frequency of clinical manifestations: Hispanic patients often present without extraocular symptoms in early phases of the disease [26], as we observed in this case, and they have globally lower rates of intertegumentary signs (between 11 and 16\%) and hearing impairment (9\%) compared to Asian patients [27]. On the other hand, neurological signs and dysacousia are quite common (74-76\%) among Japanese patients [23, 28], while intertegumentary signs are more often seen in Chinese patients (39-41\%) [29]. When looking at ocular manifestations, studies conducted on South American patients are concordant in rating a optic disc swelling occurrence significantly lower than in Asian patients (8,6\% - 9\% versus $50-73 \%$, respectively) [26-30], but they differ in rating ERD (91\% according to Sutavachtarin et al.... and $45,7 \%$ according to Giordano et al..) and anterior uveitis $(100 \%$ according to Sutavachtarin et al. and $45,1 \%$ according to Giordano et al) [26, 30]. Response to therapy and complication rates are reported to be similar among ethnic groups [26].

Such epidemiological considerations have to be taken into account when performing a differential diagnosis. In our case, also the hypothesis of a NMOSD was considered, due the history of bilateral and recurrent ONs, but diagnostic criteria where not fulfilled [10]. Also NMOSDs have higher occurrence rates in Asian patients [31]. In South America NMOSDs have been reported to have a higher prevalence in non-white populations, while MS (which is another possible misdiagnosis in VKH patients, as recently described by Algathani et al) [32] has a higher prevalence in white South Americans [33].

The present case reinforces the notion that also in Western European countries, especially in urban multiethnic populations, rare etiologies of common symptoms have to be taken into account when performing a differential diagnosis. 


\section{Abbreviations}

BCVA: best corrected visual acuity; CIS: Clinically Isolated Syndrome; CNS: Central Nervous System; CSF: cerebrospinal fluid; ERD: exudative retinal detachment; FA: Fluorescein Angiography; LP: lumbar puncture; MRI: Magnetic Resonance Imaging; MS: Multiple sclerosis; NMOSD: Neuromyelitis Optica Spectrum Disorder; OCB: oligoclonal bands; ON: optic neuritis; PS: posterior scleritis; SD-OCT: Spectral Domain Optical Coherence Tomography; VKH: Vogt-Koyanagi-Harada

\section{Funding}

The authors declare that they didn't receive any funding for this publication.

\section{Availability of data and materials}

Data sharing is not applicable to this article as no datasets were generated or analysed during the current study.

\section{Authors' contributions}

MS contributed to conception, design and draft of the manuscript for intellectual content. AP contributed to conception, design and draft of the manuscript for intellectual content. Al contributed to conception, design and draft of the manuscript for intellectual content. FV contributed to conception, design and revision of the manuscript for intellectual content. LG contributed to revision of the manuscript for intellectual content. AC contributed to revision of the manuscript for intellectual content. TC contributed to revision of the manuscript for intellectual content. MD contributed to revision of the manuscript for intellectual content. DG contributed to revision of the manuscript for intellectual content. ES contributed to revision of the manuscript for intellectual content. All authors read and approved the final manuscript.

\section{Ethics approval and consent to participate}

Not applicable.

\section{Consent for publication}

Written informed consent for publication of their clinical details and/or clinical images was obtained from the patient. A copy of the consent form is available for review by the Editor of this journal.

\section{Competing interests}

The authors declare that they have no competing interests.

\section{Publisher's Note}

Springer Nature remains neutral with regard to jurisdictional claims in published maps and institutional affiliations.

\section{Author details}

'Department of Pathophysiology and Transplantation, Neurology Unit, Dino Ferrari Center, University of Milan, Fondazione IRCCS Ca' Granda, Ospedale Maggiore Policlinico, Milan, Italy. ${ }^{2}$ Department of Clinical Sciences and Community Health, Ophtalmological Unit, University of Milan, Fondazione IRCCS Ca' Granda, Ospedale Maggiore Policlinico, Milan, Italy. ${ }^{3}$ Department of Biomedical and Clinical Science "Luigi Sacco", Eye Clinic, Luigi Sacco Hospital, University of Milan, Milan, Italy.

Received: 23 August 2017 Accepted: 14 December 2017

Published online: 28 December 2017

\section{References}

1. Sakata VM, da Silva FT, Hirata CE, de Carvalho JF, Yamamoto JH. Diagnosis and classification of VKH disease. Autoimmun Rev 2014;13:550-550.

2. Silpa-archa S, Silpa-archa N, Preble JM, Foster CS. Vogt-Koyanagi-Harada syndrome: perspectives for immunogenetics, multimodal imaging, and therapeutic options. Autoimmun Rev. 2016;15:809-19.

3. Moorthy RS, Inomata H, Rao NA. Vogt-Koyanagi-Harada syndrome. Surv Ophthalmol. 1995;39(4):265-92.

4. Abad S, Monnet D, Caillat-Zucman S, Mrejen S, Blanche P, Chalumeau M, Mouthon L, Dhote R, Brézin AP. Characteristics of Vogt-Koyanagi-Harada disease in a French cohort: ethnicity, systemic manifestations, and HLA genotype data. Ocul Immunol Inflamm. 2008;16(1-2):3-8.
5. Lavezzo MM, et al. Vogt-Koyanagi-Harada disease: review of a rare autoimmune disease targeting antigens of melanocytes. Orphanet Journal of Rare Diseases. 2016;11:29.

6. Greco A, Fusconi M, Gallo A, Turchetta R, Marinelli C, Macri GF, De Virgilio A, de Vincentiis M. Vogt-Koyanagi-Harada syndrome. Autoimmun Rev. 2013;12:1033-8.

7. Read RW, Holland GN, Rao NA, Tabbara KF, Ohno S, Arellanes-Garcia L, Pivetti-Pezzi P, Tessler HH, Usui M. Revised diagnostic criteria for VKH disease: report of an international committee on nomenclature. American Journal of Ophtalmology. 2001;131(5):647-52.

8. Toosy AT, Mason DF, Miller DH. Optic neuritis. Lancet Neurol. 2014; 13(1):83-99.

9. Gonzalez-Gonzalez LA, Molina-Prat N, Doctor P, Tauber J, Sainz de la Maza M, Foster CS. Clinical features and presentation of posterior scleritis: a report of 31 cases. Ocul Immunol Inflamm. 2014:22(3):203-7.

10. Wingerchuk DM, Banwell B, Bennett $J$, et al. International consensus diagnostic criteria for neuromyelitis optica spectrum disorders. Neurology. 2015;85:177-89.

11. Kawaguchi T, Horie S, Bouchenaki N, Ohno-Matsui K, Mochizuki M, Herbort CP. Suboptimal therapy controls clinically apparent disease but not subclinical progression of Vogt-Koyanagi-Harada disease. International Ophtalmology. 2010;30:41-50.

12. Fardeau C, Tran TH, Gharbi B, Cassoux N, Bodaghi B, LeHoang P. Retinal fluorescein and indocyanine green angiography and optical coherence tomography in successive stages of Vogt-Koyanagi-Harada disease. International Ophtalmology. 2007;27(2-3):163-72.

13. Nakai K, Gomi F, Ikuno Y, Yasuno Y, Nouchi T, Ohguro N, Nishida K. Choroidal observations in Vogt-koyanagi-Harada disease using highpenetration optical coherence tomography. Graefes Arch Clin Exp Ophthalmol. 2012;250(7):1089-95.

14. Cuevas M, De-la-Torre A, Córdoba A. Bilateral iris depigmentation and ocula Hypotony as end-stage manifestations of untreated Vogt-Koyanagi-Harada disease. Ocul Immunol Inflamm. 2017:1-6.

15. Rathinam SR, Namperumalsamy P. Global variation and pattern changes in epidemiology of uveitis. Indian J Ophthalmol. 2007;55(3):173-83.

16. Mercanti A, Parolini B, Bonora A, Lequaglie Q, Tomazzoli L. Epidemiology of endogenous uveitis in north-eastern Italy. Analysis of 655 new cases. Acta Ophtalmologica. Scandinavica. 2001;79(1):64-8.

17. Cimino L, Aldigeri R, Salvarani C, Zotti CA, Boiardi L, Parmeggiani M, Casali B, Cappuccini L. The causes of uveitis in a referral centre of northern Italy. Int Ophthalmol. 2010;30(5):521-9.

18. Chams $\mathrm{H}$, et al. Epidemiology and prevalence of uveitis: review of literature. Iranian. J Ophthalmol. 2009;21(4):4-16.

19. Yang $P$, Zhang Z, Zhou H, Li B, Huang X, Gao Y, Zhu L, Ren Y, Klooster J, Kijlstra A. Clinical patterns and characteristics of uveitis in a tertiary center for uveitis in China. Curr Eye Res. 2005;30(11):943-8.

20. Wakabayashi T, Morimura Y, Miyamoto Y, Okada AA. Changing patterns of intraocular inflammatory disease in Japan. Ocul Immunol Inflamm. 2003; 11(4):277-86.

21. Iwata D, Mizuuchi K, Aoki K, Horie Y, Kase S, Namba K, Ohno S, Ishida S, Kitaichi N. Serial frequencies and clinical features of uveitis in Hokkaido, Japan. Ocul Immunol Inflamm. 2016:1-4.

22. Ohguro N, Sonoda KH, Takeuchi M, Matsumura M, Mochizuki M. The 2009 prospective multi-center epidemiologic survey of uveitis in Japan. Jpn J Ophthalmol. 2012 Sep;56(5):432-5.

23. Takahashi $\mathrm{R}$, et al. Uveitis incidence in Jichi Medical University hospital, Japan, during 2011-2015. Clin Ophthalmol. 2017;11:1151-6.

24. Liberman P, Gauro F, Berger O, Urzua CA. Causes of uveitis in a tertiary Center in Chile: a cross-sectional retrospective review. Ocul Immunol Inflamm. 2014:1-7.

25. Llorenç $V$, et al. Epidemiology of uveitis in a western urban multiethnic population. The challenge of globalization. Acta Ophthalmol. 2015;93(6): $561-7$

26. Sukavatcharin S, et al. Vogt-Koyanagi-Harada disease in Hispanic patients. Int Ophthalmol. 2007;27:143-8.

27. Hedayaftar et al. The spectrum of Vogt-Koyanagi-Harada disease in Iran. Int Ophtalmol. 2017.

28. Sasamoto Y, Ohno S, Matsuda H. Studies on corticosteroid therapy in Vogt-Koyanagi-Harada disease. Ophthalmologica. 1990;201(3):162-7.

29. Chee SP, Jap A, Bacsal K. Spectrum of Vogt-Koyanagi-Harada disease in Singapore. Int Ophthalmol. 2007;27(2-3):137-42. 
30. Giordano VE, Schlaen A, Couto C, et al. Spectrum and visual outcomes of Vogt-Koyanagi-Harada disease in Argentina. Int J Ophthalmol. 2017; 10(1):98-102.

31. Jacob A, et al. Current concept of neuromyelitis optica (NMO) and NMO spectrum disorders. J Neurol Neurosurg Psychiatry. 2013;84(8):922-30.

32. Algathani $\mathrm{H}$, et al. Vogt Koyanagi Harada syndrome mimicking multiple sclerosis: a case report and review of the literature. Mult Scler Relat Disord. 2017:12:44-8.

33. Papais-Alvarenga RM, et al. Central nervous system idiopathic inflammatory demyelinating disorders in south Americans: a descriptive, multicenter, cross-sectional study. PLoS One. 2015;10(7):e0127757.

Submit your next manuscript to BioMed Central and we will help you at every step:

- We accept pre-submission inquiries

- Our selector tool helps you to find the most relevant journal

- We provide round the clock customer support

- Convenient online submission

- Thorough peer review

- Inclusion in PubMed and all major indexing services

- Maximum visibility for your research

Submit your manuscript at www.biomedcentral.com/submit 